\title{
A Novel Approach for Congestion Control in War State Battle Field Using Cloud Sensor for Collision Detection and Prevention
}

\author{
Kapil Kumar and Pankaj Deep Kaur \\ Department of Computer Science \& Engineering \\ Guru Nanak Dev University, Regional Campus Jalandhar, INDIA \\ er_kapilkumar@yahoo.com,pankajdeepkaur@gmail.com
}

\begin{abstract}
War State Battle Field is the basement of the military action. Battle Field is very significant for commanders to considerate and make full use of it in decision-making. This is basically depend on the vehicular traffic control in war state battle field. In this the tanks represents a vechicle. A tanks traffic on war state battle field is a vital problem and is seemly a major pretend to conclusion makers. In this research paper we will try to bring the scenario of battle field arena where the traffic consists of the vehicles called tanks. So the main focus in this research is put on the collision detection of the tanks among each other using cloud sensor by controlling the congestion in the battlefield.
\end{abstract}

Keywords: War State Battle Field, cloud sensor, cloud computing, congestion control, collision detection \& prevention

\section{Introduction}

The Cloud computing is very famous in recent years. Many connected computers distributed over a network at the same time in cloud computing. It is combination of Grid Computing and Cluster Computing [1]. Cloud computing provide services, shared resources or common infrastructure on demand through internet. Service provider provides the facilities on pay per use policy. Cloud computing has been evolved as the future generations computing paradigm. Customer can use storage space, processing capabilities, servers, operating system and application development environments. User can scale up and down the resources in an instant (timely) and on-demand manner in cloud [2]. Cloud computing allows the systems and users to use Platform as a Service (PaaS), Infrastructure as a Service (IaaS), Software as a Service (SaaS) [3]. Based on resource ownership, Cloud computing comes in three forms: public clouds, private clouds and hybrid clouds [4].

In this paper traffic over-crowding is a critical trouble in many urban centres across the universe. The existing methods are not accurate in terms of performance and cost for traffic management and control. Urban region have a more problem of traffic crushes, particularly when a bit of conjunctions take into thoughtfulness. [5]. The traffic signal is typically controlled by a controller inside a cabinet climbed on a concrete grid. Some electro-mechanical controllers are still in use [6]. Traffic control will turn a real significant topic in the hereafter when the number of road user's increases. There are several models for traffic simulation. Huge amount of traffic cause waiting and accidents. Due to heavy traffic emergency vehicles face adversities.

The traffic is not only the traffic which in considered to be on roads consisting cars, trucks bikes etc. The traffic can be in other means too e.g. air traffic where the aeroplanes and helicopters are considered to mean of communication, army vehicles traffic when going to wars etc. There are various number of traffic systems that can be considered for our research. 
But in this research we won't be considering the normal traffic of which we were talking about earlier. In this research we will try to bring the scenario of battle field arena where the traffic consists of the vehicles called tanks. The tanks in the battlefield are considered to be in motion in one direction one after the other reaching the battlefield area and shelling from the present motioned place to the other side. So the main focus in this research is put on the collision detection of the tanks among each other by controlling the congestion in the battlefield by bringing the concept of collision detection and avoidance through the algorithm which takes the speed factor as the major priority. Not only the collision is detected and avoided also the whole battlefield scenario is brought in light where tanks are shelling too.

In Battlefield Monitoring System [7] Cloud sensors and moving tanks communicate with each other and the moving tanks pass the message to fusion centre. The cloud sensors and moving tanks are powered devices. The simulation is created using the java code.

\section{Literature Survey}

2.1 The distributed treatment of traffic information collection system is realized. In [8] paper author discussed the essential troubles in the effectuation and referred settlement schemes. This project used in working client's power, net analysis sites planning and time synchronization among nodes.

2.2 Self-scheduling collects incoming vehicles into critical clusters. Author proposes [9] unfolded conclusion insurances that besides integrate look-ahead of upcoming vehicle platoons. The simulation effect show that the gain of this access is simple queue solving. The formation of "green waves" vehicles run through the road network without halting and amending overall traffic runs.

2.3 The Intelligent Traffic Control Unit focuses on three areas-Ambulance, Priority vehicles and Density control [10]. In Ambulances radio frequency identification concept is applied to fix the Ambulances track Green. The outcomes distinctly state that gamiest priority is granted to the ambulance. Secondly in priority vehicles infrared transmitter and receiver are used to make the vehicle track Green. In the third part IR and photodiodes are used in the line of sight to detect the density at the traffic signal.

2.4 This theme [11] suggests a method for accurately calculating the routine of vehicles on a route at daylight. The running aims are evoked from a frame-differencing algorithm and the data from grain unit members. The algorithm acts well below hard road traffic conditions such as traces, flora and big trucks. The most significant trinket of the aimed method is the vestiges handling utilizing sole strength of $\mathrm{B} \& \mathrm{~W}$ icons and top hat shifts.

2.5 This system based on UML. Author provides this proficiency for manipulating the traffic in main road network utilizing signs [12]. These signs are mechanically moderated aside sensors. To afford well advancement to vehicles through the road network these detectors coordinates the operation of the traffic signals in the entire area. The signal timing varies throughout the day while coordinating all the signals. It withdraws the addiction on less spoiled schemes on sign designs.

2.6 Author presented a vehicle sensing and active traffic signal time handling is used in priority based traffic light controller system [13]. The project is also designed to follow international standards for traffic light operations and control over multiple intersections. Both single and multiple intersections are dynamically adaptive to traffic conditions in these techniques.

2.7 In [14] context author presents an approach for evaluating the best energy motes for energy consumption in Battlefield Monitoring System using simulation along with 
three modes i.e. transmit mode, receive mode and idle mode. Also it evaluates the best routing protocol among which performs best in that energy motes.

\section{Pseudo Codes for Congestion Control in War State Battle Field}

\subsection{Procedure to Process Vehicle Node Information at Server Side.}

Define List VL for Vehicles V

Define List NL for Vehicle V where NL is neighbours list

While true

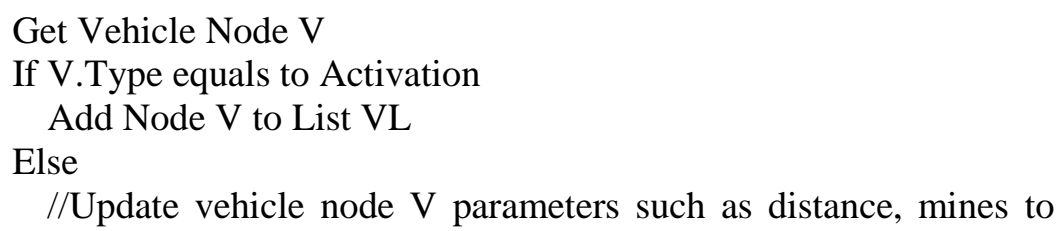

list VL

$$
\begin{aligned}
& \text { UpdateVechile(V) } \\
& \text { //Get neighbors list for node V } \\
& \mathrm{NL}=\text { GetNeighbors(V) } \\
& \text { Send list of neighbors to node V } \\
& \text { End If }
\end{aligned}
$$

End while

\subsection{Procedure to Update Vehicle Information}

Set flag found $=$ false

Set Updating Vehicle information to U

Set new templist of vehicles to empty

For each vehicle V from List VL

$$
\begin{aligned}
& \mathrm{V}=\mathrm{VL} . \text { Node } \\
& \text { If U.vehicleID == V.vehicleID) } \\
& \text { V.distance = U.distance } \\
& \text { V.mines = U.mines } \\
& \text { Add node V to templist } \\
& \text { Set flag found = true } \\
& \text { Else } \\
& \quad \text { Add node V to templist } \\
& \text { End If }
\end{aligned}
$$

End For

If flag found $==$ false

End If

Add new node $\mathrm{U}$ to templist

$\mathrm{VL}=$ templist

\subsection{Procedure to Find Neighbors for Vehicle U}

Set list of neighbors NL for node U

Set flag neighbor $=$ false

Set totalDistance $=10000 / /$ It may vary as per choice in meters

Set collisionDistance $=10 / /$ it may vary as per choice in meters

Set neighborDistance $=50 / /$ it may vary as per choice in meters

Set distance D1, D2, D3 to 0

For each node $\mathrm{V}$ in list NL

Get node $\mathrm{V}$ from list NL

If V.vehicleID != U.vehicleID 
neighbor tank $\mathrm{U}$

$$
\begin{aligned}
& \text { D1 }=\text { totalDistance }- \text { U.distance } \\
& \text { D2 }=\text { totalDistance }- \text { V.remDistance } \\
& \text { D3 }=\text { D1 }- \text { D2 } \\
& \text { If D3 }<0
\end{aligned}
$$$$
\mathrm{D} 3=(-1) * \mathrm{D} 3 / / \text { make D3 positive value }
$$

End If

If $(\mathrm{d} 3>$ collisionDistance And $\mathrm{d} 3<$ neighborDistance)

Set flag neighbor $=$ true

Add node $\mathrm{V}$ to $\mathrm{NL}$

Else If ( $\mathrm{d} 3<$ collisionDistance)

Set flag neighbor $=$ true Add node $\mathrm{V}$ to NL

//protect from collision between two vehicles

End If

PreventCollsion $(\mathrm{U}, \mathrm{V})$

If (neighbor $==$ true And U.mines $==0$ And V.mines $>0$ )

//Protect tank U by V

ProtectTank $(\mathrm{U}, \mathrm{V}) / /$ this procedure provide cover to

End If

End If

End For

\subsection{Procedure to Protect collision between Two Tanks}

PreventCollision( Tank U, Tank V)

If (U.remDistance > V.remDistance)

SpeedUp(V)

Else

SpeedDown $(\mathrm{U})$

SpeedUp(U)

End If

SpeedDown(V)

\subsection{Procedure to Change Speed of Tank}

Set toggle operation of speed for toggle $=\mathrm{Up}$

While true

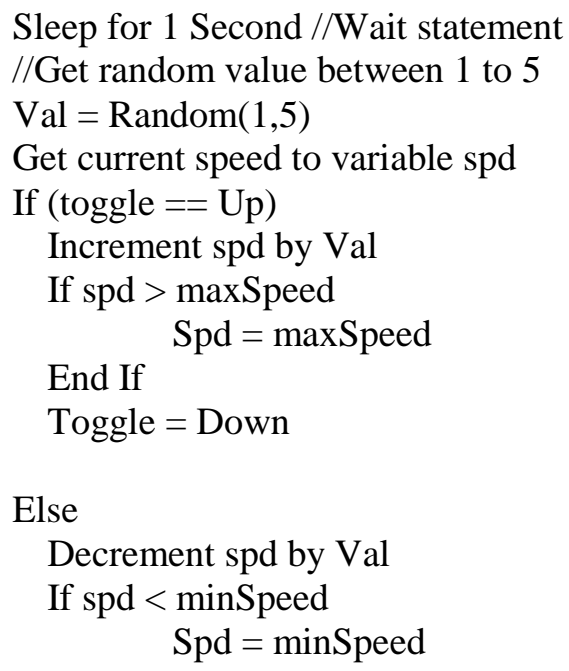




\section{End If \\ Toggle $=\mathrm{Up}$}

End If

End while

\subsection{Procedure to Speed Up Tank}

$/ /$ Get random value between 1 to 5

$\mathrm{Val}=\operatorname{Random}(1,5)$

Get current speed to variable spd

Increment spd by Val

If $\mathrm{spd}>$ max Speed

End If

$$
\text { Spd }=\text { maxSpeed }
$$

\subsection{Procedure to Speed Down Tank}

//Get random value between 1 to 5

$\mathrm{Val}=\operatorname{Random}(1,5)$

Get current speed to variable spd

Decrement spd by Val

If $\mathrm{spd}>$ minSpeed

End If

$$
\text { Spd }=\operatorname{minSpeed}
$$

\subsection{Procedure to Shoot Mines Randomly for Tank V}

While V.mines $>0$

$$
\begin{aligned}
& \text { Val = Random }(1,10) \\
& \text { Sleep for value Val } \\
& \text { Decrement value for V.mines }
\end{aligned}
$$

End While

\section{Experimental Setup}

\subsection{War State Battle Field Services}

The main objective of the proposed system is area wide management of tanks (traffic) flow aiming to maximize tanks throughput and safety of all tanks participants. It is realized by a distributed cloud sensor system based on cloud computing. The system operates by means of interaction between its components, called cloud services, using cloud server architecture. Services required for the system to function have been termed core services:

(i) Sensor Service: A Sensor Service (SS) provides data about the current road situation, such as cameras and in-road induction loop. It is a wrapper for these devices. The SS is based on associated Sensor Node. Sensor Node is able to determine the geographical position of detected objects.

(ii) Neighbour Detection Service: Neighbour Detection Service improves the routing performance in tanks, especially in networks with moderate or high mobility. In neighbour detection scheme adopts explicit handshake mechanism to reduce the latency.

(iii) Collision Prevention Service: Collision avoidance is a fundamental problem in navigation. When the tanks (Vehicle) are at risk of a collision, the system determines appropriate steering motions for both tanks at each time step, so that they can 
cooperatively change position to avoid collisions and return to their original position when the risk is averted.

(iv) Mines Cover Service: In this scenario mines represents bombs. When one tank finishes his mines then the other tank protect this tank.

(v) Distance Service: Distance is a numerical description of how far apart objects are. In most cases, "distance from A to B" is interchangeable with "distance between B and $\mathrm{A} "$. In this scenario the distance between tank $\mathrm{t} 1$ from tank $\mathrm{t} 2$ is $100 \mathrm{~m}$.

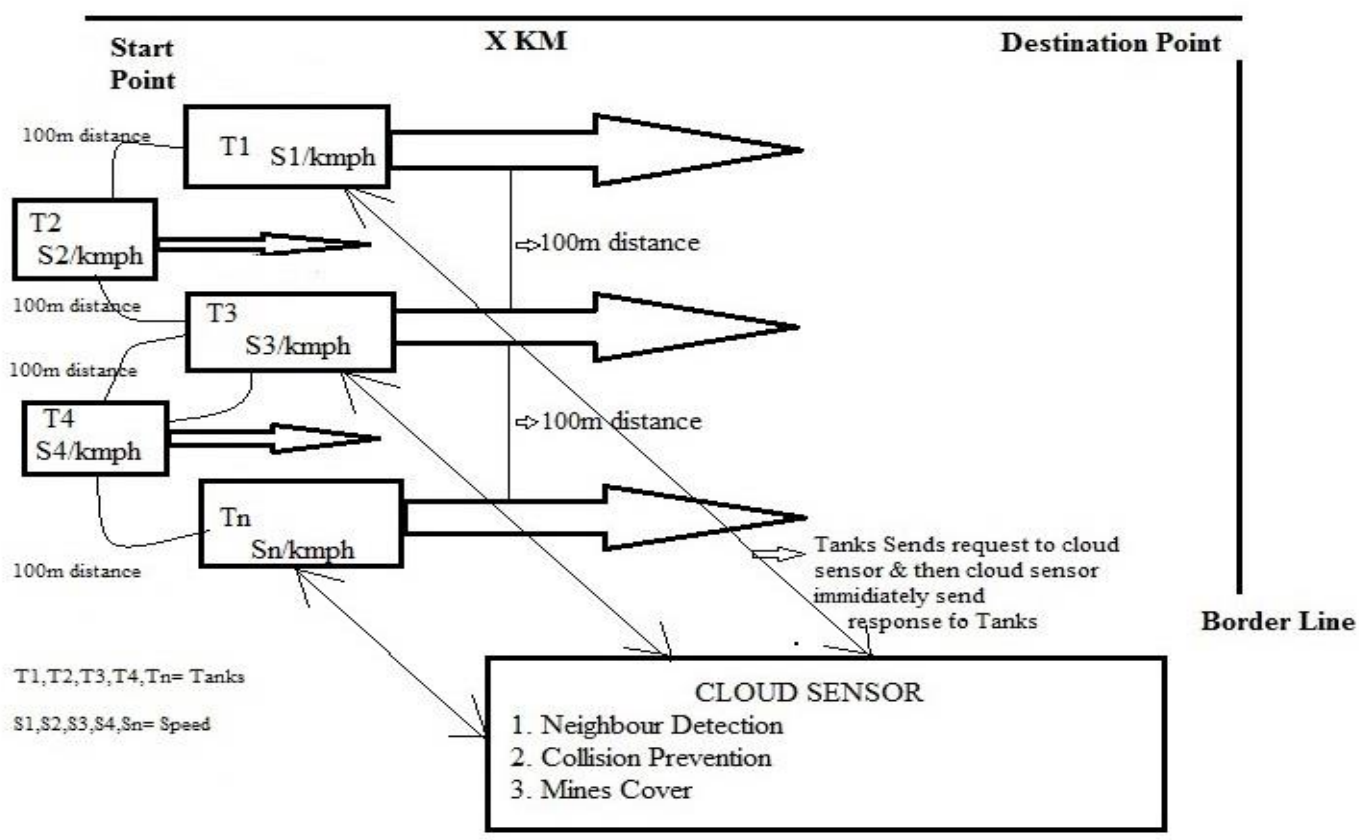

Figure 1. Scenario for Collision Detection \& Prevention in War State Battle Field

\section{Simulation and Results of War State Battle Field Using Sensor Cloud}

The above discussed benchmark algorithms were optimized using congestion control algorithms in a language java. The screen short is placed in the following figures and results of the parameters were available with collision detection and prevention algorithm in subsequent table $1,2 \& 3$.

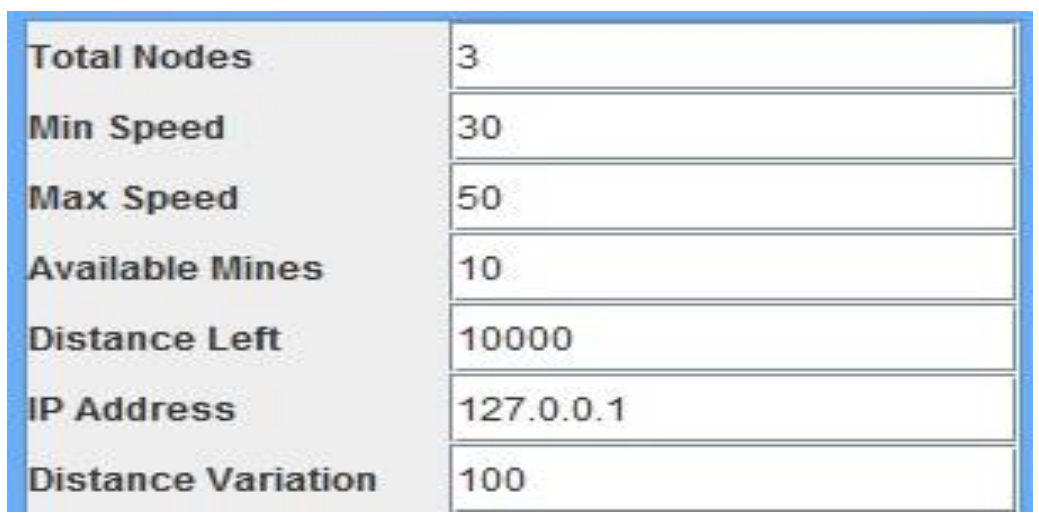

Figure 2. Optimization Parameters 
(A)

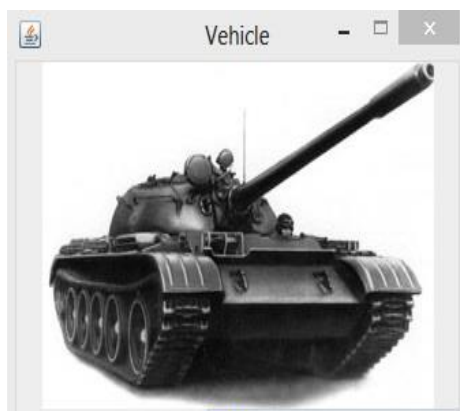

Vehicle Speed (KMPH) 37

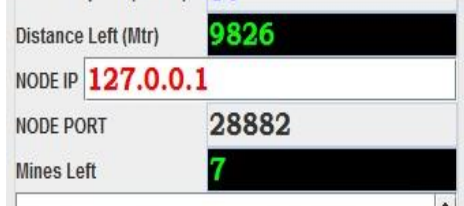

Neighbour Vehicles are running 2365125431
(B)

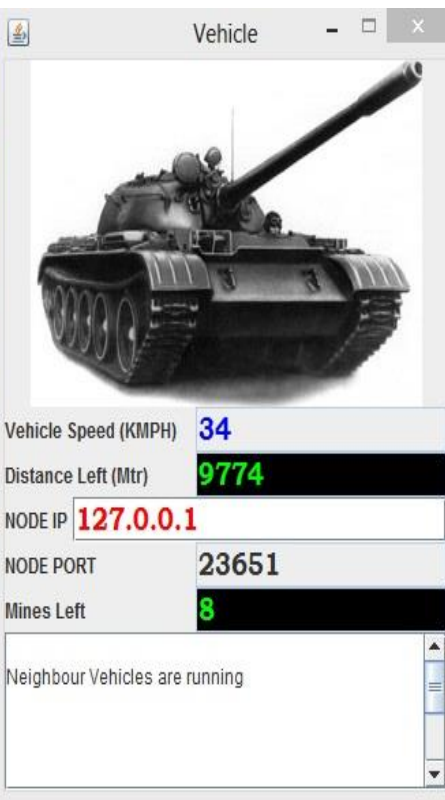

(C)

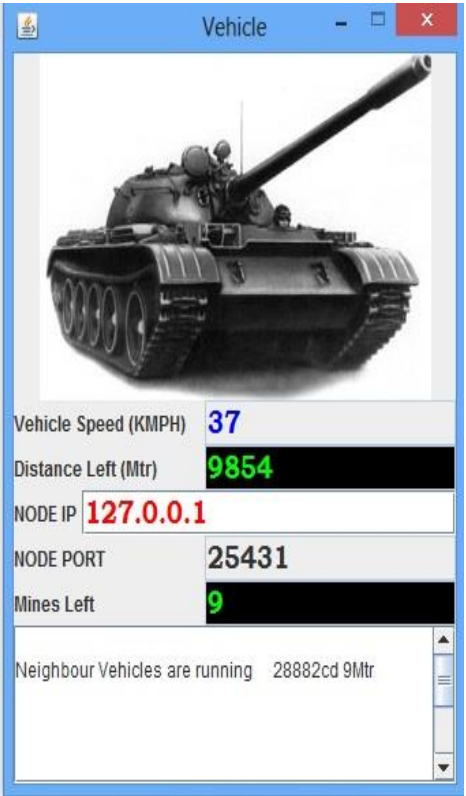

Figure 3. Three Tanks (A,B,C) Moving using Above Parameters

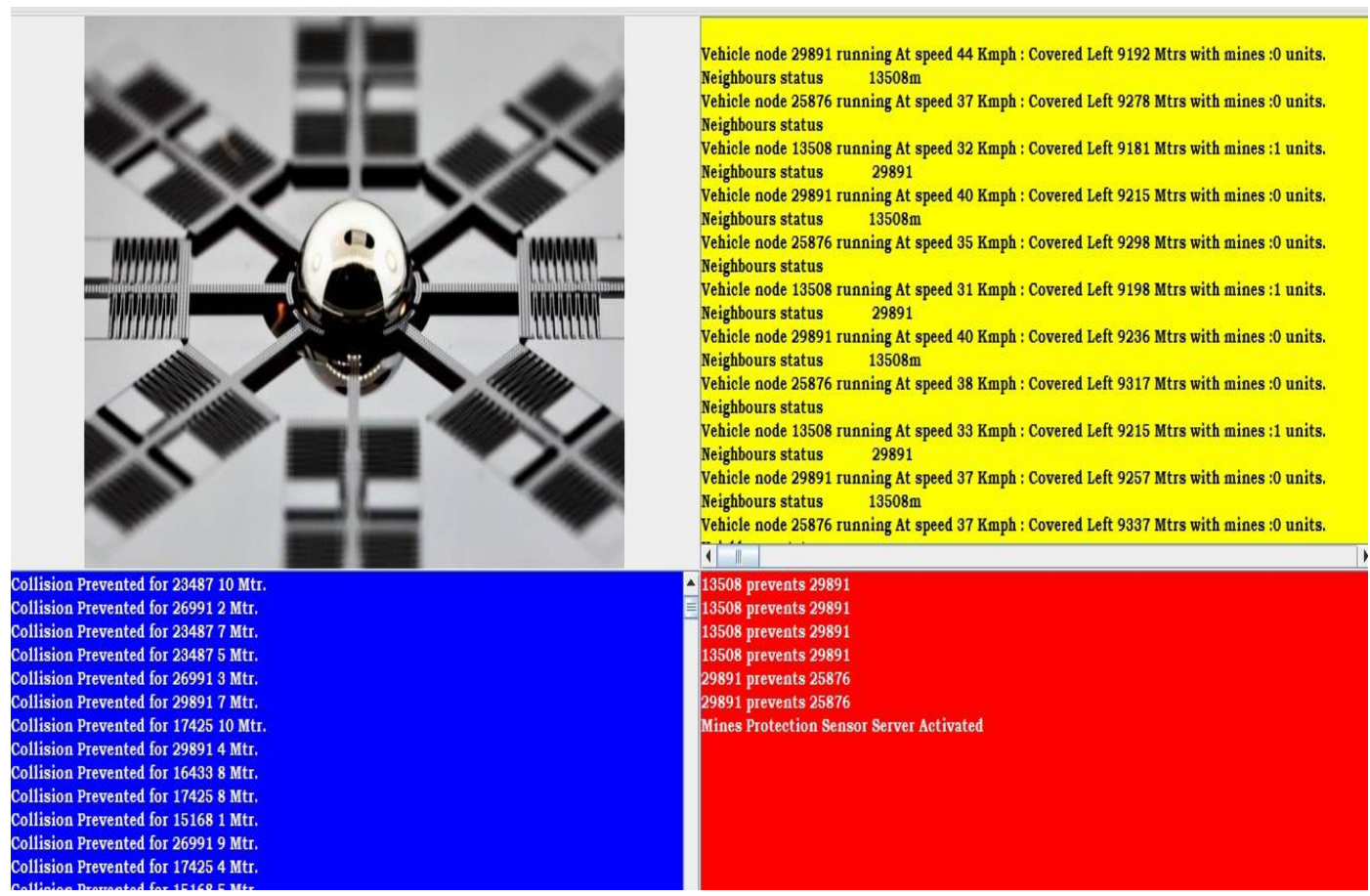

Figure 4. Optimization Results 
Table 1 and 2. Results Obtained by using Collision \& Mines Cover Parameters

\begin{tabular}{|c|c|c|c|}
\hline nodeid & collfrom & nodeid & minescover . \\
\hline 11318 & $13732 \mathrm{~cd} \mathrm{OM}$ & 14723 & $2771211899 m$ \\
\hline 11318 & $13732 \mathrm{cu} 2 \mathrm{M}$ & 14723 & $27712 \mathrm{cu} 10 \mathrm{Mtr}$ \\
\hline 11318 & $13732 \mathrm{cu} 6 \mathrm{M}$ & 14723 & 27712cu 9Mtr \\
\hline 11318 & $13732 \mathrm{cu} \mathrm{9M}$ & 18687 & $21026 \mathrm{~m}$ \\
\hline 11838 & $1432726951 \mathrm{~cd}$ & 20514 & $12953 \mathrm{~m}$ \\
\hline 11838 & $1432726951 \mathrm{~cd}$ & 20514 & $12953 \mathrm{~m}$ \\
\hline 11838 & 26951cu 2Mtr & 22422 & 14197cu 1Mtr I \\
\hline 11838 & 26951cu 6Mtr & 22422 & 14197cu 1Mtr । \\
\hline 11838 & 26951cu 9Mtr & 22422 & 14197cu 1Mtr I \\
\hline 11838 & $14327 \mathrm{~cd} 7 \mathrm{Mtr}$ & 22422 & $14197 \mathrm{~cd}$ OMtr I \\
\hline 11838 & 14327cu 2Mtr & 22422 & $14197 \mathrm{~cd} 2 \mathrm{Mtr}$ । \\
\hline 11899 & $14723 \mathrm{~cd} 2 \mathrm{Mtr}$ & 22422 & $14197 \mathrm{~cd} 1 \mathrm{Mtr}$ । \\
\hline 11899 & $14723 \mathrm{~cd} 2 \mathrm{Mtr}$ & 22422 & 14197cu 1Mtr I \\
\hline 11899 & $14723 \mathrm{~cd} \mathrm{3Mtr}$ & 22422 & 14197cu 3Mtr I \\
\hline 11899 & $14723 \mathrm{~cd} 3 \mathrm{Mtr}$ & 22422 & 14197cu 5Mtr I \\
\hline 11899 & $14723 \mathrm{~cd} 4 \mathrm{Mtr}$ & 23722 & $21162 \mathrm{~m}$ \\
\hline 11899 & $14723 \mathrm{~cd} 4 \mathrm{Mtr}$ & 27712 & $14723 \mathrm{~cd} 3 \mathrm{Mtr} \mathrm{r}$ \\
\hline 11899 & $14723 \mathrm{~cd} 6 \mathrm{Mtr}$ & 27712 & $14723 \mathrm{~cd} 3 \mathrm{Mtr}$ r \\
\hline 11899 & $14723 \mathrm{~cd} 6 \mathrm{Mtr}$ & 27712 & $14723 \mathrm{cu} 1 \mathrm{Mtr} r$ \\
\hline 11899 & $14723 \mathrm{~cd} 7 \mathrm{Mtr}$ & 27712 & 14723cu 1Mtr r \\
\hline 11899 & $14723 \mathrm{~cd} 7 \mathrm{Mtr}$ & 27712 & 14723cu 5Mtr r \\
\hline
\end{tabular}

Table 2. Results Obtained by using Simulation Parameters

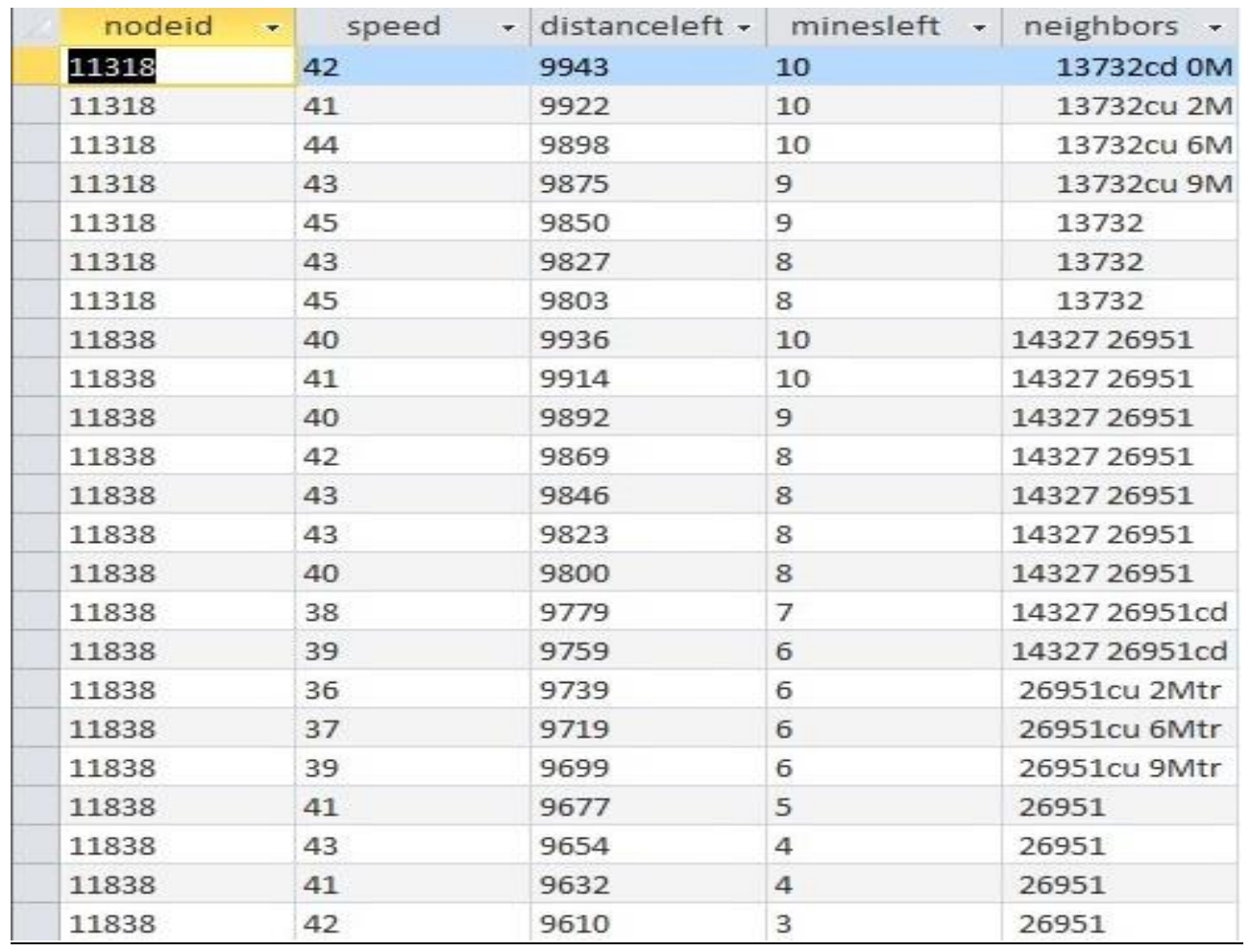




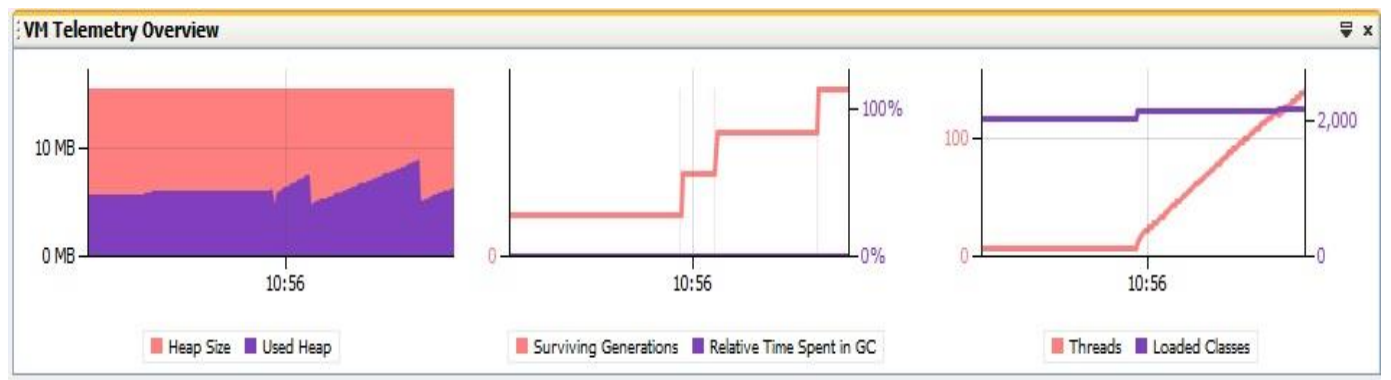

Figure 5. Graphical Representation of the Simulation Results

\section{Conclusion}

In this paper we have implemented War State Battle Field Using Cloud Sensor for Collision Detection and Prevention using Java programming in real time. This system controls the change of tanks speed at intersection points \& control the collision between two tanks. The use of this approach makes it economical, reliable and provides tanks range for communication. The system proposed in this thesis is both cost efficient in terms of communication and computation. Mathematical congestion analysis of the proposed traffic control system has been done that exhibits that the congestion control in battlefield system are secure against all traffic control and therefore can be implemented in practical scenarios. We can establish inter vehicular communication which is not present in current work instead it is to be via RSU and Cloud Servers together.

\section{References}

[1] C. Germain-Renaud and O. Rana, "The convergence of clouds, grids, and autonomics", IEEE Internet Computing, vol. 13, no. 6, (2009), p. 9.

[2] H. Alhakami, H. Aldabbas and T. Alwada, "Comparison between cloud and grid computing: review paper", International journal on cloud computing: services and architecture (IJCCSA), vol. 2, no. 4, (2012) August, pp. 1-21.

[3] H. T. Dinh, C. Lee, D. Niyato, and P. Wang, "A Survey of Mobile Cloud Computing: Architecture, Applications, and Approaches, Wireless Communications and Mobile Computing-Wiley Online Library, (2011).

[4] P. Kaur and I. Chana, "Unfolding the Distributed Computing Paradigms", 2010 International Conference on Advances in Computer EngineeringBangalore, India June 20-June 21ISBN: 978-0-7695-4058-0.

[5] C. Kim and Y.-S. Hong, "Traffic Signal Using Smart Agent System", American Journal of Applied Sciences, (2008), pp. 1487-1493.

[6] Legon-Okponglo, "Design and Development of Micro-Controller Based Traffic System Using Image Processing Techniques", University of Ghana, published in ICAST, 2012 IEEE $4^{\text {th }}$ International Conference.

[7] N. Lanjwar and D. Nitnaware, "Performance Analysis of Routing Protocols for Battlefield monitoring System", International Journal of Scientific Engineering and Technology www.ijset.com , (2012) April.

[8] X. Chen, J. Zhang, S. Qian and P. Xu, "Applied Research on Traffic Information Collection Based on Wireless Sensor Networks", Elsevier 2012 International Conference on Future Electrical Power and Energy Systems.

[9] X.-F. Xie, G. J. Barlow, S. F. Smith, and Z. B. Rubinstein, "Platoon-Based Self-Scheduling for RealTime Traffic Signal Control", IEEE International Conference on Intelligent Transportation Systems (ITSC), Washington, DC, USA, (2011).

[10] S. Jaiswal and T. Agarwal, A. Singh and Lakshita, "Intelligent Traffic Control Unit", International Journal of Electrical, Electronics ISSN No. (Online): 2277-2626 and Computer Engineering, vol. 2, no. $2,(\mathbf{2 0 1 3})$, pp. 66-72.

[11] P. F. Alcantarilla, M. A. Sotelo and L. M. Bergasa, "Automatic Daytime Road Traffic Control and Monitoring System", Intelligent Transportation Systems, 2008, ITSC 2008, 11th International IEEE Conference on (2008 ) 12-15 October, pp. 944-949.

[12] K. Ranjini, A. Kanthimathi and Y. Yasmine, "Design of Adaptive Road Traffic Control System through Unified Modeling Language", International Journal of Computer Applications (0975 - 8887) vol. 14, no. 7, (2011) February. 
[13] K. R. Shruthi and K. Vinodha, "Priority Based Traffic Lights Controller Using Wireless Sensor Networks", International Journal of Electronics, Signals, and Systems (IJESS) ISSN: 2231- 5969, vol. 1, no. $4,(\mathbf{2 0 1 2})$

[14] R. Bagga(saini), M. Bala and V. Sahni, "Energy Models with Different Routing Protocols in BMS Using Qualnet Simulator", International Journal of Advanced Research in Computer Science and Software Engineering ISSN: 2277 128X, vol. 4, no. 11, (2014), November.

\section{Author}

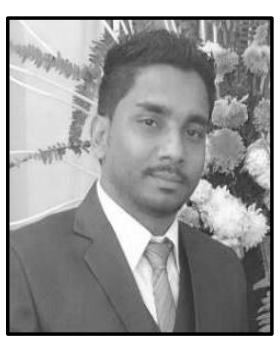

Kapil Kumar, he was born on March 41993 in Nakodar city District Jalandhar Punjab India. Completed his B.Tech (CSE) from CTIT Shahpur Jalandhar in year 2013 and Completed M.Tech in computer Science and Engineering GNDU Regional Campus Jalandhar, Punjab in year 2015. Area of interest in technology is Cloud Computing, Traffic Control of Cloud Computing, Sensor Cloud. Many research papers have been published of the author in the different conferences like IEEE, International Conferences and different Journals. Looking forward to go for Doctorate in the same field to continue his research. 